\title{
GUARANTEED COST CONTROL OF UNCERTAIN LINEAR SYSTEMS VIA DISCRETE-TIME VARIABLE STRUCTURE CONTROL
}

\author{
Ming-Chang Pai \\ pmc@nkc.edu.tw
}

Follow this and additional works at: https://jmstt.ntou.edu.tw/journal

Part of the Electrical and Computer Engineering Commons

\section{Recommended Citation}

Pai, Ming-Chang (2008) "GUARANTEED COST CONTROL OF UNCERTAIN LINEAR SYSTEMS VIA DISCRETE-TIME VARIABLE STRUCTURE CONTROL," Journal of Marine Science and Technology. Vol. 16: Iss. 1, Article 9. DOI: 10.51400/2709-6998.1999

Available at: https://jmstt.ntou.edu.tw/journal/vol16/iss1/9

This Research Article is brought to you for free and open access by Journal of Marine Science and Technology. It has been accepted for inclusion in Journal of Marine Science and Technology by an authorized editor of Journal of Marine Science and Technology. 


\title{
GUARANTEED COST CONTROL OF UNCERTAIN LINEAR SYSTEMS VIA DISCRETE-TIME VARIABLE STRUCTURE CONTROL
}

\author{
Ming-Chang Pai*
}

Key words: guaranteed cost control, discrete-time variable structure control, chattering phenomenon, linear matrix inequality.

\begin{abstract}
This paper presents a design of realizing the guaranteed cost control problem for uncertain linear systems via discrete-time variable structure control (VSC) scheme. The uncertainties in the system are assumed to be norm-bounded and time-varying. Based on the discrete-time VSC technique, the proposed controller can drive the system into a pre-specified switching surface to obtain the desired dynamic performance and the chattering phenomenon is eliminated. Sufficient conditions for the existence of guaranteed cost control are presented, and the problem of designing guaranteed cost control is converted to a convex optimization problem with linear matrix inequality (LMI) constraints. Finally, simulation results demonstrate the efficacy of the proposed control methodology.
\end{abstract}

\section{INTRODUCTION}

Variable structure control (VSC) has attractive features such as fast response, good transient performance, insensitiveness to the matching parameter uncertainties and external disturbances $[3,5,13,23]$ so that VSC is an effective robust control approach for uncertain systems. In practice, using computers or DSP chips to implement the controller becomes more and more important nowadays, and discrete-time VSC has gained more and more attractive attention recently. Different from continuous-time VSC, the motion of a discrete-time VSC system can approach the switching surface but cannot stay on it in practice. Therefore, only the quasi-sliding mode is ensured [9,16,21]. Several design methods for discrete-time VSC have been proposed in the literature $[6,7,9,10,12,16,17,20-22,26]$.

On the other hand, guaranteed cost control of uncertain linear systems has received much attention over the last few years.

Paper submitted 01/25/07; accepted 04/17/07. Author for correspondence: Ming-Chang Pai (e-mail: pmc@nkc.edu.tw).

*Department of Automation Engineering, Nan Kai Institute of Technology, Tsao-Tun, Taiwan, R.O.C.
Guaranteed cost control is firstly presented by Chang and Peng [2]. Its objective is to design a control system which is not only stable but also guarantees an adequate level of performance. Based on this idea, many significant results have been proposed for the continuous-time case $[18,24]$ and for the discrete-time case $[4,15,19,25]$. However, to the best of our knowledge, it seems that guaranteed cost control via discrete-time VSC has not been studied extensively. This has motivated our research.

The purpose of this paper is to realize the guaranteed cost control problem for uncertain linear systems based on discrete-time VSC scheme. In this proposed scheme, it possesses the properties of guaranteed cost control and VSC. Existence conditions for guaranteed cost control are derived via Lyapunov theory and linear matrix inequality (LMI) approach [1], and a convex optimization problem which is utilized to obtain guaranteed cost control is presented. The on-line disturbance rejection rule discussed by Su et al. [22] is applied to replace the conventional complex estimation methods and the chattering phenomenon is eliminated. Finally, a numerical simulation is given to illustrate the effectiveness of the proposed design approach. The proposed method has the following attractive features: 1) the order of the motion equation in the sliding mode is equal to the order of the original system, rather than reduced by the number of dimension of the control input. 2) the robustness of the system can be guaranteed throughout the entire response of the system starting from the initial time instance. 3) the control design is rather straightforward and the guaranteed cost control is achieved. 4) the discrete-time VSC needs not be of switching type and the chattering phenomenon is eliminated.

The remainder of this paper is organized as follows. Section 2 briefly states problem formulation. Section 3 provides the proposed discrete-time VSC scheme to realize the guaranteed cost control problem for uncertain linear systems. Section 4 presents results from numerical simulations. Finally, a conclusion is provided in section 5 .

\section{PROBLEM FORMULATION}

Consider a class of uncertain linear continuous-time systems represented by:

$$
\dot{x}(t)=(A+\Delta A(t)) x(t)+(B+\Delta B(t)) u(t)+f(t)
$$


where $x(t) \in R^{n}$ is the state, $u(t) \in R^{m}$ is the control input, $f(t) \in R^{l}$ is the external disturbance, $A$ and $B$ are constant matrices of appropriate dimensions. The terms $\Delta A(t)$ and $\Delta B(t)$ represent unknown time-varying parameter uncertainties of the matrices $A$ and $B$, respectively. For system (1), the following assumptions are assumed to be valid:

Assumption 1. The pair $(A, B)$ is completely controllable.

Assumption 2. There exist unknown time-varying matrix functions of appropriate dimension $D(t), L(t)$ and $H(t)$ such that $\Delta A(t)=B D(t), \Delta B(t)=B L(t)$ and $f(t)=B H(t)$. These conditions are so-called matching conditions [5].

With assumption 2, the system (1) can be rewritten as

$$
\dot{x}(t)=A x(t)+B u(t)+B d(t)
$$

where $d(t)$ is constructed as $d(t) \stackrel{\Delta}{=} D(t) x(t)+L(t) u(t)+H(t)$. Note that $d(t)$ includes the parameter uncertainties $\Delta A(t)$, $\Delta B(t)$ and the external disturbance $f(t)$. For this reason, we will call $d(t)$ as the generalized disturbance and assume $d(t)$ is bounded and smooth in the remaining of this paper.

The discrete-time representation of the system (2) with sample and hold process is given by

$$
x(k+1)=\Phi x(k)+\Gamma u(k)+\Gamma d(k)
$$

where the sampling time is $T, \Phi=e^{A T}$ and $\Gamma=\int_{0}^{T} e^{A \tau} d \tau B$. This discrete-time model is an $O\left(T^{2}\right)$ approximated model [26].

Associated with the system (3) is the cost function

$$
J=\sum_{k=0}^{\infty} x^{T}(k) Q x(k)
$$

where $Q>0$ is a given weighting matrix.

The main objective of this paper is to find a discrete-time variable structure controller such that the states of the uncertain discrete-time system (3) will asymptotically approach to zero even in the presence of parameter uncertainties and external disturbances, and the closed-loop value of the cost function (4) satisfies $J \leq J^{*}, J^{*}$ is some specified constant.

\section{Definition 1}

The magnitude of a variable v is said to be $O\left(T^{r}\right)$ if and only if $\lim _{T \rightarrow 0} \frac{v}{T^{r}} \neq 0$ and $\lim _{T \rightarrow 0} \frac{v}{T^{r-1}}=0$ where $r$ is an integer.

\section{Definition 2}

For the uncertain system (3) and the cost function (4), if there exist a control law $u^{*}(k)$ and a positive scalar $J^{*}$ such that the closed-loop system (3) with the control law $u^{*}(k)$ is asymptotically stable and the closed-loop value of the cost function (4) satisfies $J \leq J^{*}$, then $J^{*}$ is said to be a guaranteed cost and $u^{*}(k)$ is said to be a guaranteed cost control law of the uncertain system (3) and the cost function (4).

\section{MAIN RESULTS}

\section{Design of Switching Surfaces}

In this paper, the switching function is defined as

$$
\sigma(k)=G x(k)-G \sum_{i=0}^{k-1}(\Phi+\Gamma K) x(i), \sigma(0)=G x(0)
$$

where $G \in R^{m \times n}$ is chosen such that $G \Gamma$ is nonsingular and $K \in R^{m \times n}$ is designed later such that the uncertain discrete-time system (3) in the quasi-sliding mode is asymptotically stable and the closed-loop value of the cost function (4) satisfies $J \leq J^{*}$.

\section{Theorem 1}

Consider the uncertain discrete-time system (3) with the cost function (4) and the switching function (5). If there exists a symmetric positive-definite matrix $P \in R^{n \times n}$ such that the following inequality is satisfied

$$
\Phi_{c}^{T} P \Phi_{c}-P+Q<0
$$

where $\Phi_{c}=\Phi+\Gamma(G \Gamma)^{-1} G+\Gamma K$, then the uncertain discrete-time system (3) in the quasi-sliding mode is asymptotically stable. Furthermore, the corresponding value of the cost function (4) satisfies the bound

$$
J \leq x^{T}(0) \operatorname{Px}(0)
$$

where $x(0)$ is the initial state of the system (3).

\section{Proof:}

Using the concept of equivalent control, the equivalent control $u_{e q}(k)$ can be found by assuming [7]

$$
\Delta \sigma(k+1)=\sigma(k+1)-\sigma(k)=0
$$

Using (8), (5) and (3), the equivalent control $u_{e q}(k)$ can be expressed as

$$
u_{e q}(k)=K x(k)+(G \Gamma)^{-1} G x(k)-(G \Gamma)^{-1} G \Gamma d(k)
$$

where $G \Gamma$ is assumed to be nonsingular.

Substituting (9) into (3), the dynamic equation of the system (3) in the quasi-sliding mode can be obtained as

$$
x(k+1)=\Phi_{c} x(k)
$$

where $\Phi_{c}$ is defined in (6).

Next, suppose there exists a symmetric positive definite matrix $P$ such that matrix inequality (6) holds, then the Lyapunov function candidate

$$
V(k)=x^{T}(k) P x(k)
$$

is positive definite. The corresponding Lyapunov difference along the trajectory of the quasi-sliding mode dynamics (10) is 
given by

$$
\begin{gathered}
\Delta V(k)=V(k+1)-V(k) \\
=x^{T}(k)\left(\Phi_{c}^{T} P \Phi_{c}\right) x(k)-x^{T}(k) P x(k)
\end{gathered}
$$

By taking into account of (6), we have

$$
\Delta V(k)<-x^{T}(k) Q x(k)<0
$$

for any nonzero $x(k) \in R^{n}$. It follows from Lyapunov stability theory that the quasi-sliding mode dynamic of the uncertain discrete-time system (3) is asymptotically stable. Furthermore, from (13) we have

$$
x^{T}(k) Q x(k)<-\Delta V(k)
$$

Summing both sides of (14) from 0 to $\infty$ and using the system stability yield

$$
\sum_{k=0}^{\infty} x^{T}(k) Q x(k) \leq x^{T}(0) \operatorname{Px}(0)
$$

Thus, $J \leq x^{T}(0) P x(0)$. The proof is completed.

From (7), it shows that $x^{T}(0) P x(0)$ provides an upper bound on the cost function (4). In particular, note that $\operatorname{tr} x^{T}(0) P x(0)=\operatorname{tr} P x(0) x^{T}(0)$ has the same form as the $H_{2}$ cost in standard LQR theory. Hence, we replace $x(0) x^{T}(0)$ by $\Theta \Theta^{T}$ where $\Theta=x(0)$ and the guaranteed cost can be expressed as

$$
J^{*}=\operatorname{tr} P \Theta \Theta^{T}
$$

Next, we will show the sufficient condition (6) in Theorem 1 is equivalent to the feasibility of LMI.

\section{Theorem 2}

Consider the uncertain discrete-time system (3) with the cost function (4) and the switching function (5). The system (3) in the quasi-sliding mode is asymptotically stable if there exist a matrix $Y \in R^{m \times n}$ and a symmetric positive-definite matrix $W \in R^{n \times n}$ such that the following LMI is satisfied

$$
\left[\begin{array}{ccc}
-W & \bar{\Phi} W+\Gamma Y & 0 \\
W \bar{\Phi}^{T}+Y^{T} \Gamma^{T} & -W & W \\
0 & W & -Q^{-1}
\end{array}\right]<0
$$

where $\bar{\Phi}=\Phi+\Gamma(G \Gamma)^{-1} G$. Furthermore, the gain matrix $K$ is given by

$$
K=Y W^{-1}
$$

, and the corresponding value of the cost function (4) satisfies the bound

$$
J<\operatorname{tr}(Z)
$$

where $Z$ is such that the LMI variable $W$ satisfying (16) additionally satisfies

$$
\left[\begin{array}{cc}
-Z & \Theta^{T} \\
\Theta & -W
\end{array}\right]<0
$$

Proof:

From the Schur complement [1], the inequality (6) is equivalent to

$$
\left[\begin{array}{cc}
-P^{-1} & \Phi_{c} \\
\Phi_{c}^{T} & -P+Q
\end{array}\right]<0
$$

Pre- and post-multiplying both sides of (20) by

$$
\left[\begin{array}{cc}
I & 0 \\
0 & P^{-1}
\end{array}\right]
$$

where $I$ is the identity matrix of appropriate dimensions and letting $W=P^{-1}$ and $Y=K P^{-1}$, and applying the Schur complement again yield the matrix inequality (16).

Next, to show that $J$ satisfies the bound (18), we consider the inequality

$$
\Theta^{T} P \Theta<Z
$$

which yields (18). Now, using the Schur complement, it follows that the existence of $Z$ and $P$ satisfying (21) is equivalent to the existence of the existence of $Z$ and $W$ satisfying (19). Thus, to minimize the performance bound (18), we consider the LMI minimization problem

$$
\min _{W, Y, Z} \operatorname{tr}(Z)
$$

subject to $W, Y$ and $Z$ satisfying (16) and (19). This proof is completed.

Since the problem (22) is a convex optimization problem with LMI constrains. Therefore, the global minimum of the problem can be obtained if it is feasible, and it can be easily solved by using the LMI Toolbox of MATLAB [8].

\section{Design of Guaranteed Cost Controllers}

After designing the switching surface, the next phase is to design the control law such that quasi-sliding mode is reached and stayed thereafter.

From the discrete mode in (3), the one step delayed unknown disturbance

$$
\hat{d}(k-1) \stackrel{\Delta}{=} \Gamma d(k-1)=x(k)-\Phi x(k-1)-\Gamma u(k-1)
$$

can be calculated under condition of small $T$ and the smooth disturbance $[22,26]$, which implies that the unknown disturbance $\hat{d}(k)$ can be approximated by its one-step delayed value $\hat{d}(k-1)$.

For the uncertain discrete-time system (3), the control law is proposed as follows: 


$$
u(k)=K x(k)+(G \Gamma)^{-1} G x(k)-(G \Gamma)^{-1} G \hat{d}(k-1)
$$

where the gain matrix $K$ is given by (17).

Combining (24) and (3), the augmented system becomes

$$
\left[\begin{array}{l}
x(k+1) \\
u(k+1)
\end{array}\right]=\hat{\Phi}\left[\begin{array}{l}
x(k) \\
u(k)
\end{array}\right]+\left[\begin{array}{l}
I \\
K
\end{array}\right] \hat{d}(k-1)
$$

where

$$
\hat{\Phi}=\left[\begin{array}{cc}
\Phi & \Gamma \\
K \Phi+(G \Gamma)^{-1} G \Phi & K \Gamma+(G \Gamma)^{-1} G \Gamma
\end{array}\right]
$$

The stability of the augmented system is stated as the following lemma.

\section{Lemma 1}

The eigenvalues of $\hat{\Phi}$ are $\operatorname{eig}\left\{\Phi+\Gamma(G \Gamma)^{-1} G+\Gamma K\right\}$ and zero.

\section{Proof:}

Let $\lambda$ be an eigenvalue of $\hat{\Phi}$; then

$$
\begin{gathered}
0=\operatorname{det}\left(\lambda I_{n+m}-\hat{\Phi}\right) \\
=\operatorname{det}\left[\begin{array}{cc}
\lambda I_{n}-\Phi & -\Gamma \\
-K \Phi-(G \Gamma)^{-1} G \Phi & \lambda I_{m}-K \Gamma-(G \Gamma)^{-1} G \Gamma
\end{array}\right]
\end{gathered}
$$

Premultiplying the first $n$ rows by $-(G \Gamma)^{-1} G$ and adding to the last $m$ rows yields

$$
0=\operatorname{det}\left[\begin{array}{cc}
\lambda I_{n}-\Phi & -\Gamma \\
-K \Phi-\lambda(G \Gamma)^{-1} G & \lambda I_{m}-K \Gamma
\end{array}\right]
$$

Premultiplying the first $\mathrm{n}$ rows by $-K$ and adding to the last $m$ rows yields

$$
\begin{aligned}
& 0=\operatorname{det}\left[\begin{array}{cc}
\lambda I_{n}-\Phi & -\Gamma \\
-\lambda K-\lambda(G \Gamma)^{-1} G & \lambda I_{m}
\end{array}\right] \\
& =\lambda^{m} \operatorname{det}\left[\begin{array}{cc}
\lambda I_{n}-\Phi & -\Gamma \\
-K-(G \Gamma)^{-1} G & I_{m}
\end{array}\right]
\end{aligned}
$$

Premultiplying the last $\mathrm{m}$ rows by $\Gamma$ and adding to the first $n$ rows yields

$$
\begin{gathered}
0=\lambda^{m} \operatorname{det}\left[\begin{array}{cc}
\lambda I_{n}-\left(\Phi+\Gamma(G \Gamma)^{-1} G+\Gamma K\right) & 0 \\
-K-(G \Gamma)^{-1} G & I_{m}
\end{array}\right] \\
=\lambda^{m} \operatorname{det}\left[\lambda I_{n}-\left(\Phi+\Gamma(G \Gamma)^{-1} G+\Gamma K\right)\right]
\end{gathered}
$$

This proof is completed.

\section{Remark 1}

Using (5), (3), (23) and (25), we have

$$
\Delta \sigma(k+1)=G[\hat{d}(k)-\hat{d}(k-1)]=G \Gamma[d(k)-d(k-1)]
$$

If the disturbance $d(t)$ is bounded and smooth, $d(k)-d(k-1)$ is of $O(T)$. From the definition given in (3), the norm of $\Gamma$ is $O(T)$. Hence, $\Delta \sigma(k+1)$ has a magnitude of the order $O\left(T^{2}\right)$. From Lemma 1 and (27), we concluded that the control law (24) will drive the state to travel in the vicinity of the switching surface at each sampling instant $t=k T$.

From Theorem 2 and Lemma 1, Theorem 3 can be obtained as follows.

\section{Theorem 3}

For the uncertain discrete-time system (3) with the cost function (4) and the switching function (5), if the generalized disturbance $d(t)$ in (2) is bounded as well as smooth, and the following optimization problem

$$
\min _{W, Y, Z} \operatorname{tr}(Z)
$$

s.t.

$$
\begin{gathered}
{\left[\begin{array}{ccc}
-W & \bar{\Phi} W+\Gamma Y & 0 \\
W \bar{\Phi}^{T}+Y^{T} \Gamma^{T} & -W & W \\
0 & W & -Q^{-1}
\end{array}\right]<0} \\
{\left[\begin{array}{cc}
-Z & \Theta^{T} \\
\Theta & -W
\end{array}\right]<0}
\end{gathered}
$$

has solutions $W, Y$ and $Z$, then the control law (24) is a guaranteed cost control law which ensures the minimization of the guaranteed cost function (4) of the system (3).

The procedures of the proposed discrete-time VSC to realize the guaranteed cost control problem for uncertain linear systems are summarized as follows:

i) Choose $Q$ (Eq. 4) and $G$ (Eq. 5)

ii) Calculate $K$ (Eqs. 17 and 28).

iii) Approximate the disturbance $\hat{d}(k)$ (Eq. 23).

iv) Calculate $u(t)$ (Eq. 24).

\section{ILLUSTRATIVE EXAMPLE}

In this section, we consider a continuous-time water-quality dynamic model of the River Nile proposed in [14] without the delay terms, and the system parameter matrices are given as follows:

$$
\begin{gathered}
A=\left[\begin{array}{cc}
-1 & 1 \\
-2 & -3
\end{array}\right], B=\left[\begin{array}{cc}
1 & 0 \\
0 & 0.5
\end{array}\right], \\
\Delta A(t)=\left[\begin{array}{cc}
0.1 \sin (2 t) & -0.3 \sin (t) \\
-0.1 \sin (t) & 0.075 \sin (3 t)
\end{array}\right], \\
\Delta B(t)=\left[\begin{array}{cc}
0.25 \sin (t) & -0.15 \sin (2 t) \\
-0.15 \sin (t) & 0.05 \sin (3 t)
\end{array}\right],
\end{gathered}
$$




$$
f(t)=\left[\begin{array}{c}
0.2+0.6 \sin (t) \\
0.1+0.09 \sin (3 t)
\end{array}\right]
$$

Associated with this system is the cost function (4) in which the weight matrix is given as follows:

$$
Q=\left[\begin{array}{cc}
10 & 0 \\
0 & 10
\end{array}\right]
$$

The discrete-time transformed system (3) for each sampling time can be easily obtained by using the Matlab program function c2d [11] with sample time $T=0.01$ second and given as

$$
\Phi=\left[\begin{array}{cc}
0.99 & 0.0098 \\
-0.0196 & 0.9703
\end{array}\right], \Gamma=\left[\begin{array}{cc}
0.0099 & 0.0000 \\
-0.0001 & 0.0049
\end{array}\right]
$$

Similarly, according to assumption 2 and (3), it is easy to check that the perturbations $\Delta A(t), \Delta B(t)$ and $f(t)$ are matched and the generalized disturbance $d(k)$ is given as

$$
d(k) \stackrel{\Delta}{=} D(k) x(k)+L(k) u(k)+H(k)
$$

where

$$
\begin{gathered}
D(k)=\left[\begin{array}{cc}
0.1 \sin (2 k) & -0.3 \sin (k) \\
-0.2 \sin (k) & 0.15 \sin (3 k)
\end{array}\right], \\
L(k)=\left[\begin{array}{cc}
0.25 \sin (k) & -0.15 \sin (2 k) \\
-0.3 \sin (k) & 0.1 \sin (3 k)
\end{array}\right], \\
H(k)=\left[\begin{array}{c}
0.2+0.6 \sin (k) \\
0.1+0.18 \sin (3 k)
\end{array}\right]
\end{gathered}
$$

Applying the proposed method, the switching surface matrix is chosen as $G=\left[\begin{array}{ll}1 & 0 \\ 0 & 2\end{array}\right]$ so that $G \Gamma=\left[\begin{array}{cc}0.0099 & 0.0000 \\ -0.0002 & 0.0099\end{array}\right]$ is nonsingular. Next, we will determine gain matrix $K$ that minimizes $\operatorname{tr}(Z)$ with some constraints (28). Using the software LMI toolbox in MATLAB [8], it is found that the corresponding optimization problem (28) with $\Theta=\left[\begin{array}{ll}0.5 & 1\end{array}\right]^{T}$ is feasible, and the optimal solutions are given by

$$
W=\left[\begin{array}{cc}
0.0987 & -0.0000 \\
-0.0000 & 0.0715
\end{array}\right], Y=\left[\begin{array}{cc}
-20.8617 & 0 \\
0 & -20.8617
\end{array}\right],
$$

$$
Z=24.1174
$$

Then, the state feedback gain matrix from (17) can be obtained as $K=\left[\begin{array}{cc}-211.2813 & -0.0821 \\ -0.0821 & -291.6851\end{array}\right]$. According to Theorem 3 and (24), the optimal guaranteed cost controller is

$$
u(k)=\left[\begin{array}{cc}
-110.7821 & -0.5855 \\
1.9312 & -88.6734
\end{array}\right] x(k)
$$

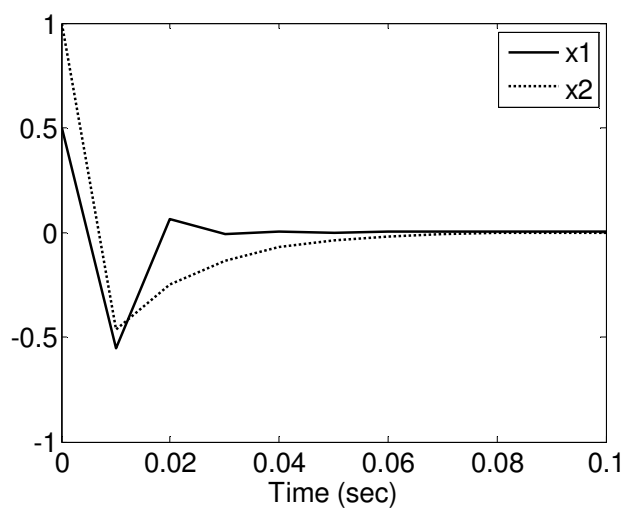

Fig. 1. System states.

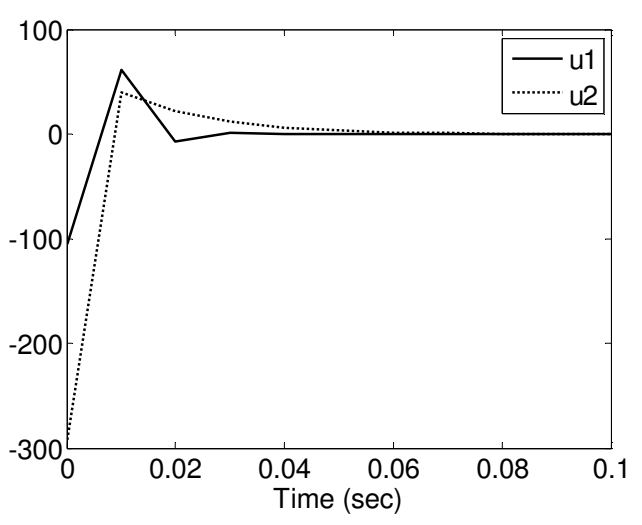

Fig. 2. Control inputs.

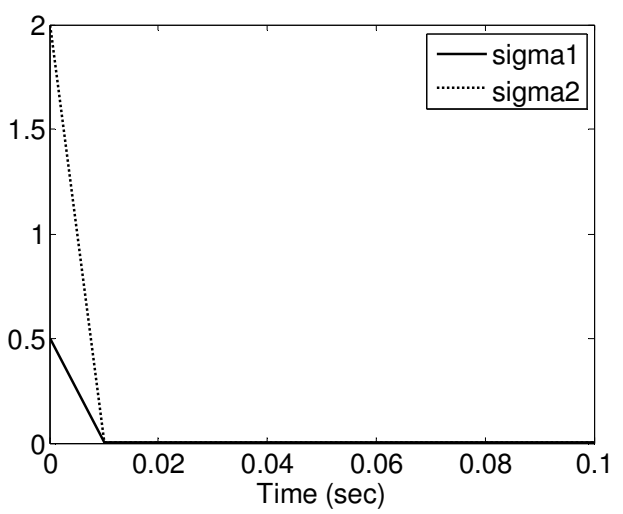

Fig. 3. Switching functions.

$$
-\left[\begin{array}{cc}
100.4992 & -0.5033 \\
2.0133 & 203.0117
\end{array}\right] \hat{d}(k-1)
$$

where $\hat{d}(k-1)$ is given by (23). The associated upper bound of the closed-loop cost value is $J^{*}=24.1174$.

With the designed parameter setting and initial condition $x(0)=\left[\begin{array}{ll}0.5 & 1\end{array}\right]^{T}$, the closed-loop dynamic responses of simu- 
lation are shown in Figs. 1-3. Fig. 1 shows the trajectories of the system. It is clear shown that the states of the system approach to zero in finite time. Fig. 2 shows the control input with reasonable magnitudes. Fig. 3 shows the switching function. It can be seen that the chattering phenomenon is eliminated.

\section{CONCLUSION}

In this paper, we have considered the guaranteed cost control problem via discrete-time VSC scheme for uncertain linear systems. This new discrete-time VSC algorithm possesses the properties of guaranteed cost control and VSC. A simple on-line disturbance estimator has been applied, and the discrete-time VSC needs not be of switching type. The existence condition for guaranteed cost control has been derived. Furthermore, the guaranteed cost control problem has been converted to a convex optimization problem with LMI constraints. The simulation results have been verified the theoretical analysis and demonstrated the effectiveness of the proposed control methodology.

\section{REFERENCES}

1. Boyd, S., Ghaoui, L. E., Feron, E. and Balakrishnan, V., Linear Matrix Inequalities in System and Control Theory, SIAM, Philadelphia (1994).

2. Chang, S. S. L. and Peng, T. K. C. "Adaptive Guaranteed Cost Control of Systems with Uncertain Parameters," IEEE Trans. Automat. Control, Vol. 17, No. 4, pp. 474-483 (1972)

3. DeCarlo, R. S., Zak, S. and Mathews, G., "Variable Structure Control of Nonlinear Multivariable Systems: A Tutorial," Proc. IEEE, Vol. 76, pp. 212-232 (1988)

4. Dong, Z. and You, Z. "Optimal Guaranteed Cost Control of A Class of Nonlinear Uncertain Discrete-time Systems: An LMI Approach," Proceeding of American Control Conference, pp. 5039-5042 (2006).

5. Drazenovic', B. "The Invariance Condition in Variable Structure Systems," Automatica, Vol. 5, pp. 287-295 (1969).

6. Eun, Y. S., Kim, J. H., Kim, K. G., and Cho, D. I., "Discrete-time Variable Structure Controller with A Decoupled Disturbance Compensator and Its Application to A CNC Servomechanism," IEEE Transaction on Control Systems Technology, Vol. 7, No. 4, pp. 414-423 (1999).

7. Furuta, K., "Sliding Mode Control of A Discrete System," System and Control Letters, Vol. 14 pp. 145-152 (1990).

8. Gahinet, P., Nemirovski, A., Laub, A. J. and Chilali, M., LMI Control Toolbox, The MathWorks Inc., Natick (1995).

9. Gao, W. B., Hung, Y., and Homaifa, A., "Discrete-Time Variable Structure
Control Systems," IEEE Trans. on Ind. Electr., Vol. 42, No. 2, pp.117-122 (1995).

10. Garcia, J. P. F., Ribeiro, J. M. S., Silva, J. J. F. and Marins, E. S., "Continuous-Time and Discrete-Time Sliding Mode Control Accomplished Using A Computer," IEE Proc.-Control Theory Appl., Vol. 152, No. 2, pp. 220-228 (2005).

11. Grace, A., Control Toolbox, The MathWorks Inc., Natick, (1993).

12. Hu, J. B., Shi, M H., and Tian, Y. C., "A New Switching Function for Variable Structure Control," Proceedings of the 5th World Congress on Intelligent Control and Automation, pp. 1163-1166 (2004).

13. Hung, J. Y., Gao, W. and Hung, J. C., "Variable Structure Control: A Survey," IEEE Trans. Ind. Electron, Vol. 40, pp. 2-22 (1993).

14. Mahmoud, M. S., "Dynamic Control of Systems with Variable State-Delay," Int. J. Robust and Nonlinear Control, Vol. 6, pp. 123-146 (1996).

15. Mahmoud, M.S. and Xie, L., "Guaranteed Cost Control of Uncertain Discrete Systems with Delays," Int. J. Control, Vol. 73, No. 2, pp. 105-114 (2000).

16. Milosavljevic, D., "General Conditions for the Existence of A Quasi-sliding Mode on the Switching Hyperplane in Discrete Variable Structure Systems," Automt. Remote Contr., Vol. 46, pp. 307-314 (1985).

17. Monsees, G. and Scherpen, J. M. A., "Adaptive Switching Gain for A Discrete-Time Sliding Mode Controller," Int. J. Control, Vol. 75, No. 4, pp. 242-251 (2002).

18. Petersen, I. R., "Guaranteed Cost LQG Control of Uncertain Linear Systems," IEE Proc. Control Theory Appl., Vol. 142, No.2, pp. 95-102 (1995).

19. Petersen, I. R., McFarlane, D. C., and Rotea, M. A., "Optimal Guaranteed Cost Control of Discrete-Time Uncertain Linear Systems," Int. J. Robust Nonlinear Control, Vol. 8, pp. 649-657 (1998).

20. Pan, Y. and Furuta, K., "Vss Controller Design for Discrete-Time System," Control-Theory and Advanced Technology, Part 1. Vol. 10, No. 4, pp. 669-687 (1994).

21. Sarpturk, S. Z., Istefanopulos, Y., and Kaynak, O., "On the Stability of Discrete-Time Sliding Mode Control Systems," IEEE Trans. Automat. Control, Vol. 32, No. 10, pp. 930-937 (1987).

22. Su, W. C., Drakunov, S. V., and Özgüner, Ü., “An O $\left(T^{2}\right)$ Boundary Layer in Sliding Mode for Sampled-Data System," IEEE Transactons on Automatic Control, Vol. 45, No. 3, pp. 482-485 (2000).

23. Utkin, V. I., "Variable Structure Systems with Sliding Modes," IEEE Trans. Automatic Control, Vol. 22, No. 2, 212-222 (1977).

24. Yu, L., "An LMI Approach to Guaranteed Cost of Linear Uncertain Time-delay Systems," Automatica, Vol. 35, pp. 1155-1159 (1999).

25. Yu, L. and Gao, F., "Optimal Guaranteed Cost Control of Discrete-Time Uncertain Systems with Both State and Input Delays," Journal of The Franklin Institute, Vol. 330, pp. 101-110 (2001).

26. Young, K. D, Utkin, V. I., and Özgüner, Ü., "A Control Engineer's Guide to Sliding Mode Control," IEEE Transactions on Control System Technology, Vol.7, No. 3, pp. 328-342 (1999). 